\title{
Post-Stall Motions Evolving Toward Chaos
}

\author{
Simone Zuccher* and Sergio De Ponte \\ Politecnico di Milano, 20158 Milano, Italy.
}

DOI: $10.2514 / 1.25389$

\begin{abstract}
The spin of an airplane occurs for angles of attack beyond stall, where nonlinear aerodynamics dominates and where complex and unpredictable behaviors might induce to question whether or not such a motion is chaotic. To find an answer to this issue, wind-tunnel tests are carried out on a model of a fighter attached by its center of gravity through an universal joint that allows only the three rotations. These degrees of freedom are analyzed according to modern techniques for the study of "supposedly chaotic data." It is found that, for increasing Reynolds number, successive bifurcations take place with a consequent more complex structure of the attractor, which reveals some features typical of quasi-periodic systems evolving toward chaos. The model is tested also in other configurations (different nose and/or leading-edge extensions, presence or absence of tail planes) so as to verify the dependence of the motion on some details. It is found that unpredictability and strong dependence on the initial conditions characterize the basic configuration, whereas a blunt nose and leading-edge extensions make the motion extremely regular. Even though the system might be on its route to chaos, a fully developed chaotic behavior is not observed.
\end{abstract}

\section{Nomenclature}

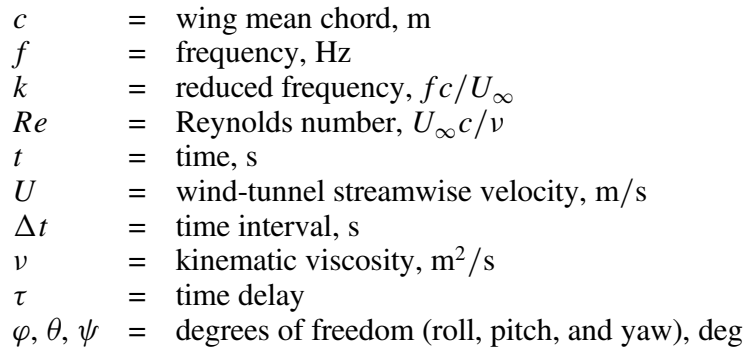

Subscript

$\infty \quad=$ asymptotic (freestream) conditions

\section{Introduction}

$\mathbf{S}_{\mathrm{f}}^{\mathrm{P}}$ PIN is an aggravated stall that results in autorotation [1]. The flight path is a downward spiral, in which the airplane descends toward the ground while rotating around a vertical axis, rolling, yawing, pitching, and sideslipping as a consequence of being at some angle of attack between stall and $90 \mathrm{deg}$ [2]. For definitions of various spin types and modes (equilibrium or steady vs oscillatory, erect vs inverted, flat vs steep, etc.) the reader is referred to standard books, e. g., [3]].

During this motion, the control surfaces fall in the large wake originating from the separated flow over the wing leading to loss of control effectiveness. The combination of separated flows, high rotational rates, and high coupling in all axes make spin one of the most complicated motions an airplane can experience. This can result in a very difficult spin recovery or, in the worst-case scenario, in fatal accidents. The latter are mainly caused by the fact that the pilot can be extremely disoriented and not able to give the required control input to bring the aircraft back to a stable trimmed state. Therefore, prediction and analysis of airplane spin characteristics together with

Received 23 May 2006; accepted for publication 20 October 2006. Copyright $\odot 2006$ by Simone Zuccher and Sergio De Ponte. Published by the American Institute of Aeronautics and Astronautics, Inc., with permission. Copies of this paper may be made for personal or internal use, on condition that the copier pay the $\$ 10.00$ per-copy fee to the Copyright Clearance Center, Inc., 222 Rosewood Drive, Danvers, MA 01923; include the code 0021-8669/ $07 \$ 10.00$ in correspondence with the CCC.

*Research Associate; currently Department of Computer Science, University of Verona, Verona; zuccher@sci.univr.it. Member AIAA

${ }^{\dagger}$ Associate Professor, Department of Aerospace Engineering, Via La Masa 34; deponte@ aero.polimi.it. recovery strategies have been of great interest since the beginning of aviation.

The analysis of spin is extremely complex, involving phenomena such as flow separation and nonlinear aerodynamics. This led to the development of highly specialized testing techniques for documenting fully developed spin characteristics [4], such as outdoor free-flight tests using radio-controlled models or tests in vertical wind tunnels. Although these techniques provide information regarding the spin behavior of an aircraft, they require special facilities and apparatus, which may not be available easily [57]. An alternative is flight testing, which can be particularly useful to analyze the characteristics of spin entry and recovery. For instance, Stough et al. [8] report some results obtained from flight tests on a low-wing general-aviation research airplane performed by changing tail configuration, mass distribution, position of center of gravity, and control inputs. However, due to the limited ability of the disoriented pilot to apply the proper spin recovery procedures (as a consequence of the high angular rates), the use of suitable automatic spin recovery schemes in flight tests is mandatory.

Because of the complications and costs of full-scale experiments and scaled models in dedicated facilities, analytical techniques based on results from conventional wind-tunnel tests were developed. These techniques rely on the fact that there exists a balance between the aerodynamic and inertial forces and moments [9]. Such methods, however, involve a significant computational effort and require repetition of wind-tunnel tests for various control settings. These problems, to a large extent, were overcome by the introduction of bifurcation and continuation methods for analyzing aircraft dynamics.

Bifurcation analysis is a popular method used to study nonlinear systems. It was first initiated in flight dynamics by Carroll and Mehra [10] and Zagayonov and Goman [11]. Goman et al. [12] present an extended review on the application of global stability and bifurcation analysis for different nonlinear flight dynamics problems such as roll coupling, stall, spin, etc. Guicheteau $[13,14]$ studied several spin motions leading to toroidal structures and chaos, and showed that results from bifurcation analysis can be used in the design of control surfaces for spin recovery. Bifurcation analysis applied to multipleattractor flight mechanics has also been considered [15].

The theoretical foundation of most contemporary continuation methods, on the other hand, can be found in the seminal work of Davidenko [16]. The method has evolved since then, but it is still a fundamental tool for constructing equilibrium curves and/or surfaces, which pose a considerable challenge and are needed for the qualitative analysis.

As far as the recovery phase of spin is concerned, some of the theoretical research was directed toward the analysis of the effect of 
geometric configuration on the early stage of recovery, such as the effects of horizontal tail configurations and inertia moments $[9,17,18]$. Functional optimization [19] and angular momentum suppression method $[20,21]$ were used to successfully determine an optimal control or a valid method for recovering from spin. In these studies, however, the efforts were directed at slowing angular rates to bring the airplane into controllable conditions and reduce the angle of attack to a region where aerodynamic controls are effective. Also, methods for full automated spin recovery have been developed so as to arrest rotation and restore prespin conditions [22].

The fact that aircraft flight dynamics is inherently nonlinear, both from the point of view of the equations of motions and with regard to the aerodynamic forces and moments, allowed the emergence of a variety of qualitative new phenomena such as chaotic dynamics.

The work in [23] investigates regions of chaotic response in the post-stall regime via bifurcation analysis. Methods of nonlinear dynamics were applied to the longitudinal motion of a vectored thrust aircraft at high angles of attack using data for the aerodynamic coefficients obtained by NASA wind-tunnel tests on the F/A-18 high-alpha research vehicle (Harv) in the post-stall regime [24]. Chaotic motion was observed at certain frequencies, whereas at other frequencies several limiting states coexist, e.g., chaotic attractor and a limit circle, or two limit cycles. Periodic bifurcations were observed also while investigating the role of thrust vectoring in spin entry and recovery [25].

Guided by these observations and by the fact that sometimes an aircraft spin is called irregular because the pilot is not able to recognize any known behavior, we performed some experiments to address the possibility that a chaotic motion may appear in spins. In fact, an aircraft has enough degrees of freedom to allow chaotic motions [26] and its dynamics is strongly nonlinear. A further motivation for a better understanding of the possible chaotic nature of spin is the design of an automatic recovery system. A tendency to chaos, in fact, means unpredictability and sensitivity to initial conditions, which raises serious questions on the possibility to control spin or to instruct the pilot on what to do to recover the airplane. Control strategies based on quasi-static aerodynamic models were quite unsuccessful [10], possibly because of the consequent quasi-static nature of the bifurcation analysis involved. Therefore, control strategies for aircraft spin recovery are necessarily complex and nonlinear [27]. A recent review on this issue can be found in [28], where the problem of spin recovery is addressed by employing nonlinear dynamic inversion techniques.

In the present study a modular model of an aircraft, which would perform the worst possible spin according to some conceptual criteria, was tested. Mounted by its center of gravity through an universal joint, it was free to rotate in a conventional wind tunnel for the required number of revolutions. The time series of the three coupled reference angles were recorded and then analyzed, employing the typical tools of nonlinear dynamics and chaos.

\section{Experimental Setup}

\section{A. Wind-Tunnel Setting}

Tests were performed in a closed-loop wind tunnel with open test section, whose usable portion is about $1.2 \mathrm{~m}$ in diameter. The flow quality, therefore, was not excellent, with a turbulence level ranging between 0.35 and $0.5 \%$. This was not a major problem for these experiments because the flow over the model during spin tests is fully turbulent and mainly separated. No effects of a possible residual swirl were observed. In fact, they would have determined a constant spin direction, regardless of the model tested. This did not occur because the spin direction changed according to the model configuration.

Because this is a conventional wind tunnel, the choice of how to anchor the model posed some issues. The first one was whether to allow all 6 degrees of freedom or only the 3 rotations. Because of the formidable complications imposed by the first option, the simplest solution was to support the model by its center of gravity, allowing only rotations and employing a sting whose axis would be aligned with the freestream flow. To assess how well this 3-degree-of-

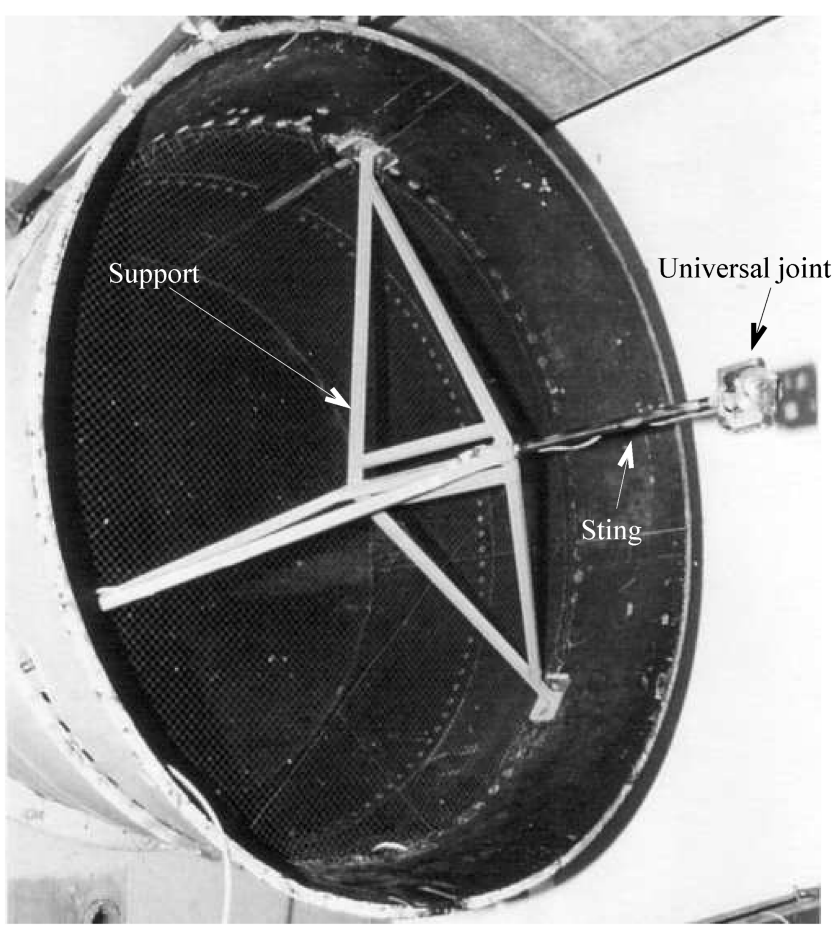

Fig. 1 Wind-tunnel test section setup, flow from right to left.

freedom gimbaled rig represents the full 6-degree-of-freedom spin motions, free-flight tests (e.g., in vertical wind tunnels) would be needed. However, for the purpose of investigating the possible chaotic nature of spin, which requires two independent spin motions, the present configuration with only 3 degrees of freedom is expected to be satisfactory. Figure 1 reports a picture of the wind-tunnel setup. A tripod is set in the divergent so as to anchor the sting to the wind tunnel. The model had to be connected to the sting by a universal joint and a hinge around the yaw axis to allow the three rotations $\varphi, \theta, \psi$. The joint is shown in Fig. 2 along with the degrees of freedom $(\psi$ denotes the yaw rotation). The model is anchored to the universal joint through four screws (see the holes on the picture) so that its lower side would be visible on Fig. 2 if bolted. Figure $\underline{3}$ reports the reference frame with the goal of showing that $\varphi$ is the rotation of plane $\beta$ with respect to plane $\alpha$, where $\alpha$ is fixed to the ground reference frame. $\theta$ is the rotation of plane $\gamma$ with respect to plane $\beta$, and $\psi$ is the yaw rotation (i.e., in the direction normal to plane $\gamma$ ). The main limitation of such a joint is that rotations $\varphi$ and $\theta$ are not complete. This was not a problem in the present experiments because the maximum angles for $\varphi$ and $\theta$ were never reached in the tests, as

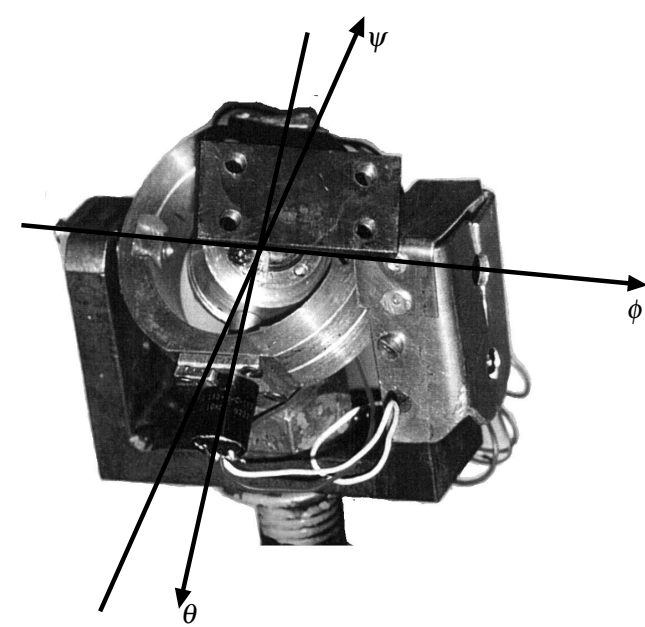

Fig. 2 Universal joint. The potentiometer that detects the rotation $\theta$ is visible. 


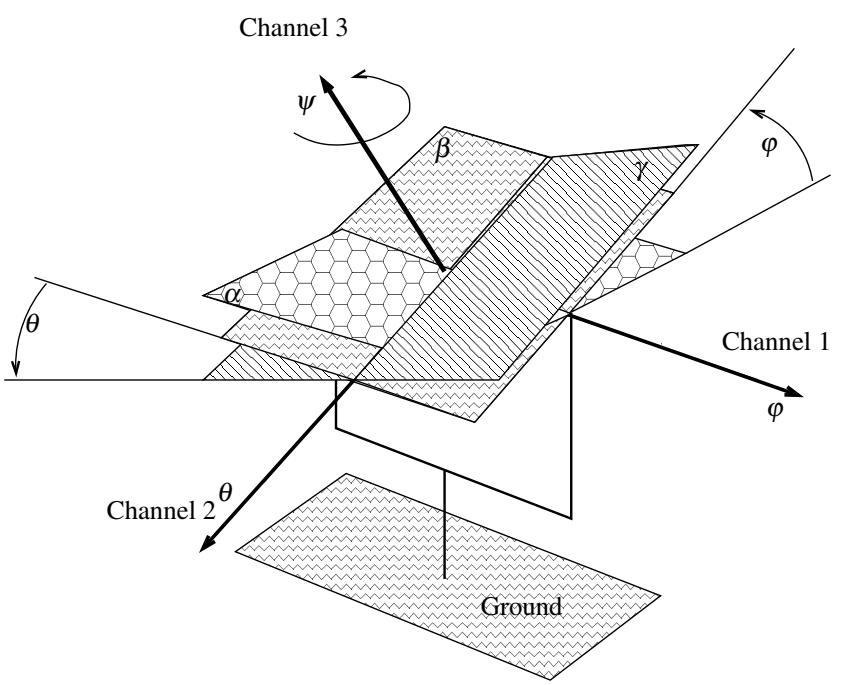

Fig. 3 Sketch of the reference frame. This is a roll-pitch-yaw sequence $(\varphi, \theta, \psi)$.

proved by the time series. Because the joint assembly is elongated in one direction and the joint is buried inside the model, these maxima for $\varphi$ and $\theta$ are functions of $\psi$. In the worst-case scenario, i.e., for $\varphi$ and $\theta$ greater than $65 \mathrm{deg}$ and for certain values of $\psi$, the model can hit the physical limits of the mechanism. However, from the plots reported in Sec. IV, devoted to the results, it can be noticed that in all tests $\varphi$ and $\theta$ were always lower than $50 \mathrm{deg}$; thus, hitting could not take place. Moreover, if any hitting occurred, it would have shown up in the time series with sharp discontinuities in the time derivatives of $\varphi$ and $\theta$. Because such discontinuities were never observed in the time series or in any plot, the possibility of the model hitting the mechanical limits of the joint during the tests is excluded.

Chronologically, the design of the joint and the selection of the way of detecting the motion were the very first steps in the design of the experiments. In fact, the size of the joint would have fixed any other model dimension because the model had to be realized to contain the whole mechanism for the detection of the rotations [29]. Knowing that frictional damping had given many problems in windtunnel tests for wing rocking, ball bearings were the first choice. The smallest size of the standard commercial bearings, therefore, provided the overall joint dimensions. For the detection of the motion many systems were considered, i.e., magnetic, resistive, and photoelectrical devices. For the sake of simplicity and for their reduced size, small stopless potentiometers (visible in Fig. 2) were chosen.

\section{B. Model Design}

The model was designed following the idea that a chaotic behavior is theoretically possible if there are two independent spin motions [30]. For our experiments, the first motion was assumed to be governed by the wing stall [31] and the second one by the asymmetry of the vortices originating from the nose of the forebody [32].

The leading-edge stall of the wing was ensured by the very bad stall properties of the five-digit NACA 23012 airfoil. The asymmetry of the roll moment induced by the wing stall provides autorotation for the initiation of spin [33]. The wing was not twisted so as to enhance these features.

The nose of the forebody, on the other hand, plays a fundamental role in the initial stages of the second type of spin. In fact, it is well known that beyond a certain angle of attack the flow over a cone is dominated by asymmetrical vortices originating in the proximity of the cone nose [34-37]. The consequent yaw moment, which depends on some apex asymmetries, provides the yaw rotation.

Also, the geometry of the tail was chosen to favor the spin motion. Among different possibilities, the " $T$ " configuration was preferred because it provides the maximum interference with the wing wake, which, in turn, causes the most irregular effects similar to the "deep stall" (or "superstall") in forward flight. From the tests it was found, a

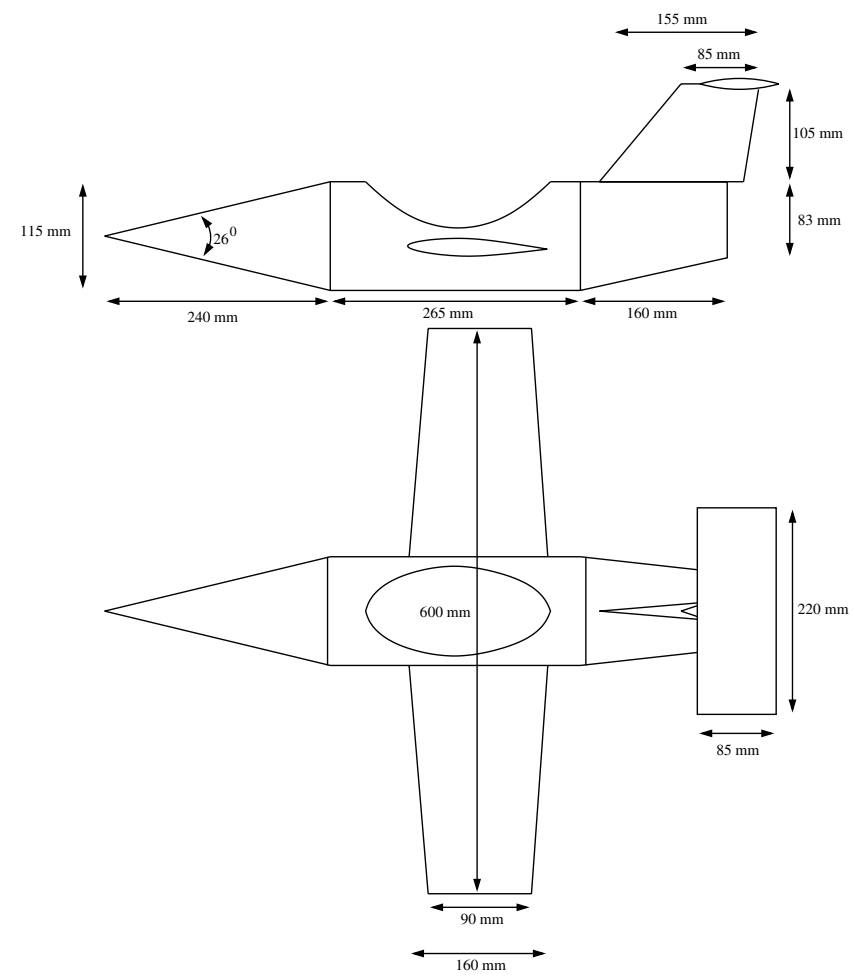

Fig. 4 Sketch of the wind-tunnel model.

posteriori, that the " $\mathrm{T}$ " tail can interact with the vortices originating from the nose of the model and determine the spin motion.

The manufacturing technology was the simplest possible, due to time and budget constraints. The main part of the fuselage was a piece of tube made of aluminum alloy with a conical sandwich aluminumcarton nose. The wing was plastic foam with a fiber-epoxy resin skin. After some efforts, everything was designed small enough to be contained in the useful part of the test section of our wind tunnel. Figure 4 reports the sketch of the model in its basic configuration (dimensions in millimeters). Additional features are as follows. Wing surface: $60,625 \mathrm{~mm}^{2}$, aspect ratio 6 , trapezoidal wing. Horizontal tail plane: surface $18,700 \mathrm{~mm}^{2}$, aspect ratio 2.75 (rectangular). Vertical tail plane: surface $12,600 \mathrm{~mm}^{2}$, sweep angle $50 \mathrm{deg}$. Figure 5 shows the picture of the model in basic configuration (as in Fig. 4), whereas Fig. 6 reports the picture of additional configurations that were tested, with blunt nose and/or leading-edge extensions (LEX). The blunt cone forming the forebody had the same height as the sharp-cone configuration and the same base radius. The frontal area of the model is about $2.33 \times 10^{-2} \mathrm{~m}^{2}$, which corresponds to a blockage of $2 \%$ (referred to the wind-tunnel section).

The model was statically stable and accurately balanced. In addition, its behavior was checked by spinning it "wind-off" so as to ensure the correct performance of the whole joint mechanism.

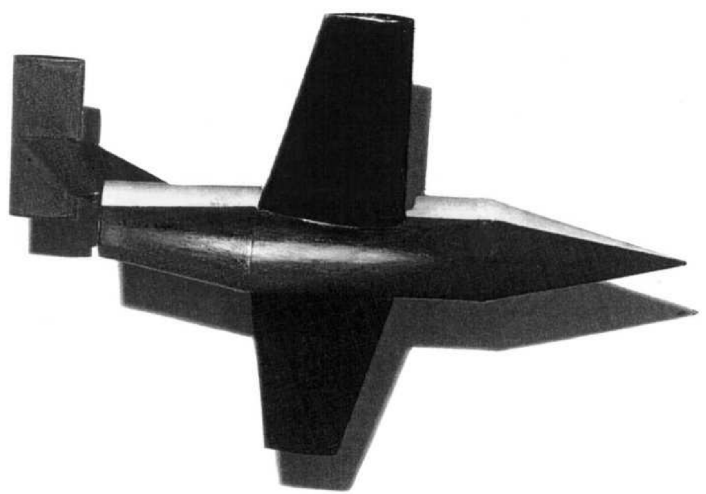

Fig. 5 Picture of the wind-tunnel model in basic configuration. 


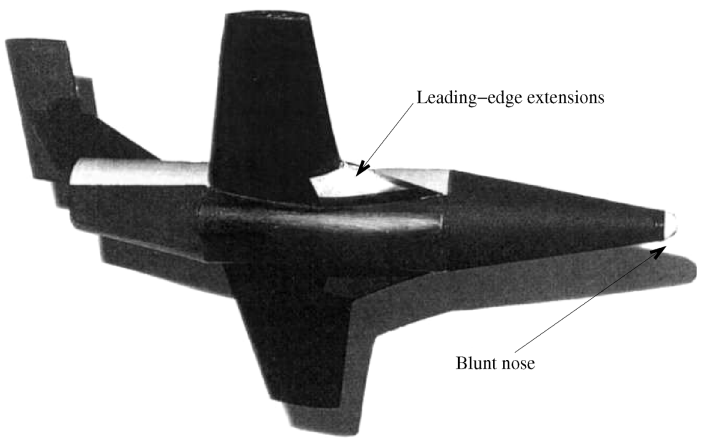

Fig. 6 Picture of the wind-tunnel model in modified configuration.

\section{Data Analysis}

\section{A. Data Reduction}

The position of the model as a function of time is uniquely determined by the time series of the three angles $\varphi, \theta$, and $\psi$ (see Figs. 2 and 3 for their definitions). These angles are obtained from the output of the potentiometers (a voltage between 0 and $10 \mathrm{~V}$ ) converted in degrees through the (linear) calibration curve. The raw voltage is sampled using a 12-bit analog-to-digital board that can operate at high frequency $(1 \mathrm{MHz})$.

The main inconvenience in the postprocessing of the data is the fact that the rotation around the yaw axis causes a discontinuity in the signal every time an entire rotation is completed. In practice, this results in a periodic discontinuity of the signal that jumps from 0 to $360 \mathrm{deg}$ every revolution. This inconvenience is removed by restoring the continuity of the data, which in turn produces a time series that looks like a periodic oscillation superimposed to a linear function of time. Instead of high-passing such a signal so as to retrieve the oscillation purged from the uniform rotation around the yaw axis, we preferred to first fit the data with a linear function and then subtract this linear dependence from the signal. The linear behavior was obtained by best-fitting the data in the sense of the mean squares. As opposed to high-passing the signal, this technique removes only the linear dependence on time, whereas all the other (low) frequencies are still present in the signal for the analysis.

\section{B. Analysis of Chaotic Data}

The analysis of "supposedly chaotic" time series is nowadays well developed [38-41] and has been employed for investigating experimental data obtained in the most diverse fields. Typically it provides $[42,43] 1)$ frequencies of motion, 2) period doubling, and 3 ) attractors (maybe strange, in some sense). The tools used to gather this information from the time series are [38-41] 1) power spectra of the signals, 2) phase portrait, 3) Poincaré maps, and 4) reconstruction of the attractor in the embedding space.

The frequency analysis (power spectrum) provides the first insights regarding the possible periodic, quasi-periodic, or chaotic nature of the signal and can easily exclude the presence of chaos. Specifically, a periodic signal is characterized by a single frequency and its harmonics, detectable in the spectrum. This is the simplest situation and can be verified, in case of multiple frequencies, by checking if the frequency ratios are rational. It goes without saying that, when dealing with experimental data, it might be sometimes difficult to prove that a number is rational because of the uncertainties in the measurement. However, in our experiments it was quite easy to discriminate between rational frequency ratios and irrational ones (see Sec. IV devoted to the results). In the latter case the signal is said quasi-periodic and the power spectrum typically becomes broader than for periodic signals. The further increase of the number of frequencies in the signal, toward a broader band, increases also the chances for the signal to be chaotic. This broadband feature, however, should not lead to the straightforward conclusion that the signal is chaotic. In fact, also completely random signals are characterized by a broadband spectrum (white noise) but are not chaotic. Therefore, once the power spectrum excludes the possibility of periodic or quasi-periodic behaviors, further investigations are needed to assess the possible chaotic nature of the data.

Phase portrait is a more sophisticated tool that consists in plotting the state variables (typically positions and velocities) as a function of time in two- or three-dimensional spaces. The phase space has usually a dimension greater than three, which implies the impossibility to visualize the complete attractor. Alternatively, the projection of the attractor on specific planes can be analyzed, but this could generate possible intersections that might not be present in the original phase space. Phase portrait is a powerful technique because it shows clearly the nature of the attractor and thus can help to visually discriminate between periodic, quasi-periodic, and chaotic data. In particular, whereas periodic or quasi-periodic systems provide a low number of trajectories (one for each frequency in the power spectrum), chaotic behaviors produce a portrait that resembles a bundle.

Poincaré maps are usually employed to "clean" the phase portrait from the overwhelming number of trajectories, while retaining the underlying information. In fact, even in the cases for which the dimension of the phase space is less than four, the phase portrait might sometimes look very confusing because of the presence of too much information (trajectories) on the plot. If $\gamma$ is the trajectory in the $n$-dimensional phase space, it is always possible to intersect it with a surface $\Sigma$. The points so obtained constitute the Poincaré section, which turns out to be a very simple plot, typically two-dimensional (to be visualized). From the spatial disposition of the points originating from the intersection with $\Sigma$ it is possible to retrieve the nature of the attractor. In particular, if there is only one point or very few points the system is periodic (single frequency or harmonics), whereas if the points on $\Sigma$ form more complex and structured plots then the system can be quasi-periodic or chaotic. If no structure can be identified and the points occupy the whole section, the signal is most likely random. Poincaré sections are particularly useful because they allow the identification of the attractor given few information (few points as opposed to full trajectories in the phase space) and can easily discriminate between random and chaotic signals because the latter have a strong spatial correlation.

Another technique for the analysis of observed chaotic data is the reconstruction of the phase space from a single time series. This would not be necessary for our experiments, because three state variables are available. However, we wanted to check if the same structure of the phase portrait could be retrieved by employing one signal alone. The "embedding" theorem, attributed to Takens [44], ensures that, if it is possible to observe (measure) a single scalar quantity $h(t)$, then the geometrical structure of the complete system can be unfolded in the space $y(t)$ reconstructed from $h(t)$ as $\boldsymbol{y}(t)=\{h(t), h(t+\tau), h(t+2 \tau), h(t+3 \tau), \ldots, h[t+(d-1) \tau]\}$, where $d$ is the dimension of the embedding space. It goes without saying that the choice of both $\tau$ and $d$ is a central issue for the reconstruction. Without entering the details, for which the reader is referred to [40], some good prescriptions should be followed for the choice of $\tau$. First, it should be a multiple of the sampling time. Second, it should not be too short, otherwise $h(t)$ and $h(t+\tau)$ would not be sufficiently independent, i.e., some time delay is needed to ensure an evolution that introduces new information. Finally, $\tau$ should not be too large because, being a chaotic system intrinsically unstable, $h(t)$ and $h(t+\tau)$ would be completely uncorrelated for a large value of $\tau$. On the other hand, the choice of $d$, which is an integer, must guarantee that the attractor is unfolded such that no unwanted overlaps occur. The latter could originate when the attractor is projected on a lower-dimension space, as it happens for the projections on a specific plane of the phase portrait. The theorem, fortunately, provides a lower limit for $d$ (sufficient condition), $d>2 d_{A}$, where $d_{A}$ is the dimension of the set on which the motion lies $\left(d_{A}\right.$ can be fractional).

\section{Results}

Preliminary tests were conducted at fixed Reynolds number $R e=U_{\infty} c / v$, where $c=0.125 \mathrm{~m}$. These tests aimed at visually inspecting the model motion and estimating the minimum value of 
Reynolds number required for the spin to occur. If the Reynolds number was not sufficient to excite the motion, an increment of $1 \mathrm{~m} / \mathrm{s}$ was applied to the freestream speed $U_{\infty}$ without stopping the tunnel. This was done only during the preliminary tests, whereas during the measurements in basic and modified configurations the wind-tunnel velocity was always restarted from $U_{\infty}=0$ and gradually increased to the target value. Sometimes an impulse was manually impressed to the model so as to force the rotation and to observe whether there was any damping or forcing effect. The model was effectively spinning, without a manual external forcing, for $R e>4 \times 10^{4}$, i.e., for freestream speeds greater than about $5 \mathrm{~m} / \mathrm{s}$, and spinning was always in the same direction for a fixed geometry. On the contrary, changes in the geometry (nose and/or LEX) caused changes in the spin direction. This confirmed what could have been supposed by common knowledge of the flow over cones, according to which any asymmetry on the cone surface would always produce the same effects, thus leading to a single direction of rotation for a given geometry.

\section{A. Basic Configuration}

All results discussed in this section refer to the model in Fig. 5. Many tests were carried out in the range of Reynolds numbers between $4 \times 10^{4}$ and $2.65 \times 10^{5}$, but here we report only the most relevant plots to describe the typical features observed.

Figure 7 refers to $R e=4.8 \times 10^{4}$ and shows the power spectrum of $\varphi$ (channel 1) as a function of the reduced frequency defined as $k=f c / U_{\infty}$. The main peak located at $k=5.3 \times 10^{-3}(0.215 \mathrm{~Hz})$

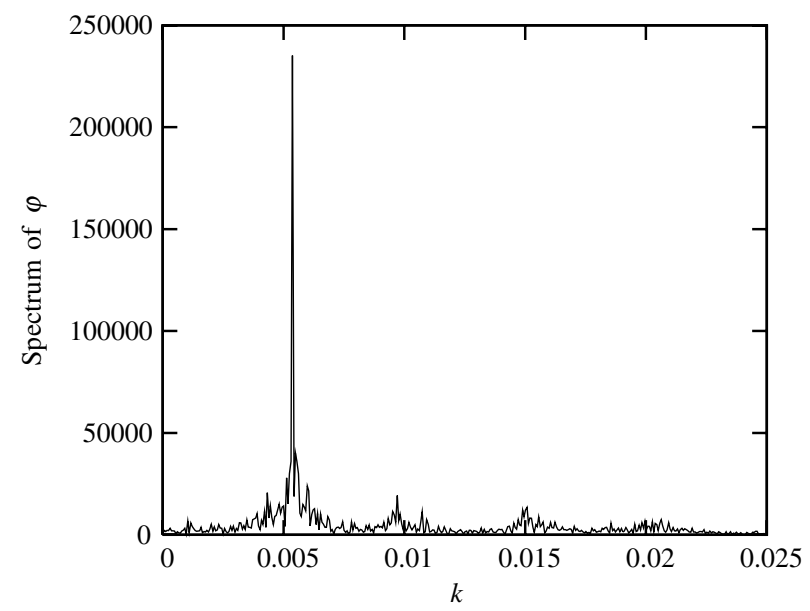

Fig. $7 R e=4.8 \times 10^{4}$. Power spectrum of $\varphi($ channel 1$)$ vs the reduced frequency $k=f c / U_{\infty}$.

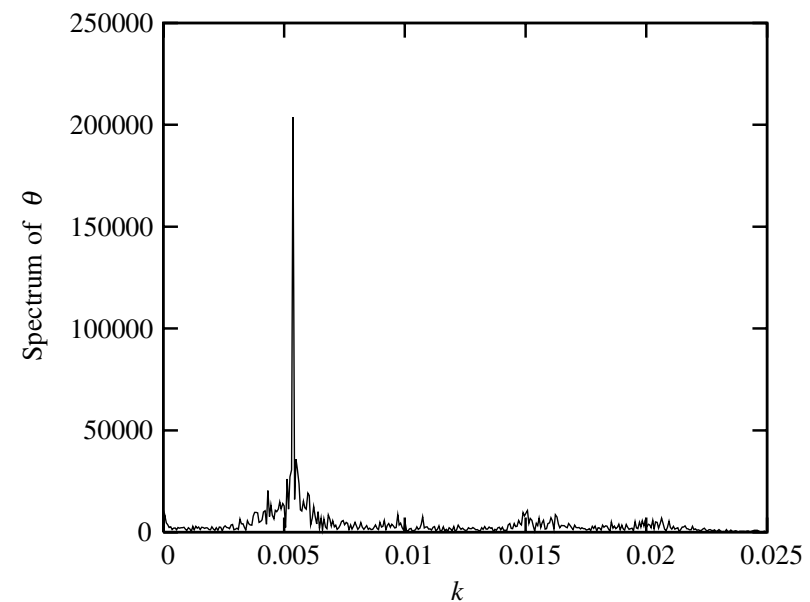

Fig. $8 R e=4.8 \times 10^{4}$. Power spectrum of $\theta($ channel 2$)$ vs the reduced frequency $k=f c / U_{\infty}$.

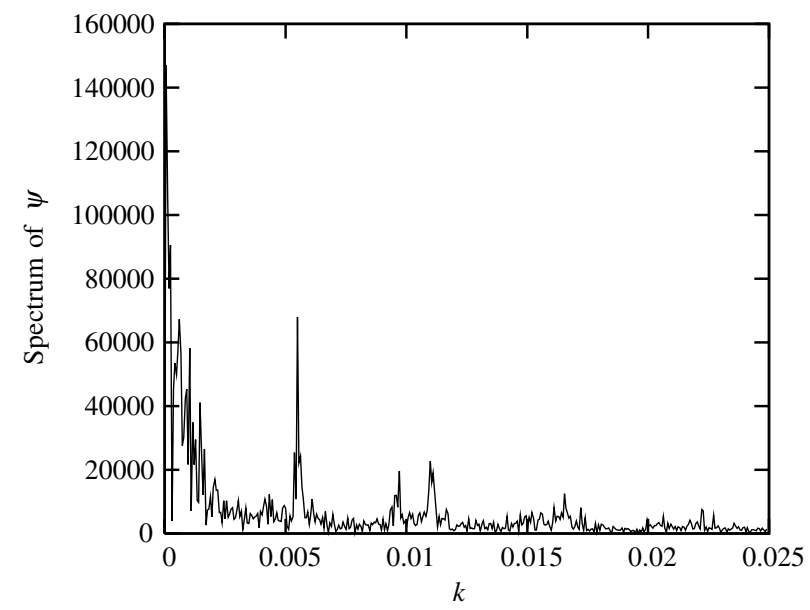

Fig. $9 R e=4.8 \times 10^{4}$. Power spectrum of $\psi($ channel 3) vs the reduced frequency $k=f c / U_{\infty}$.

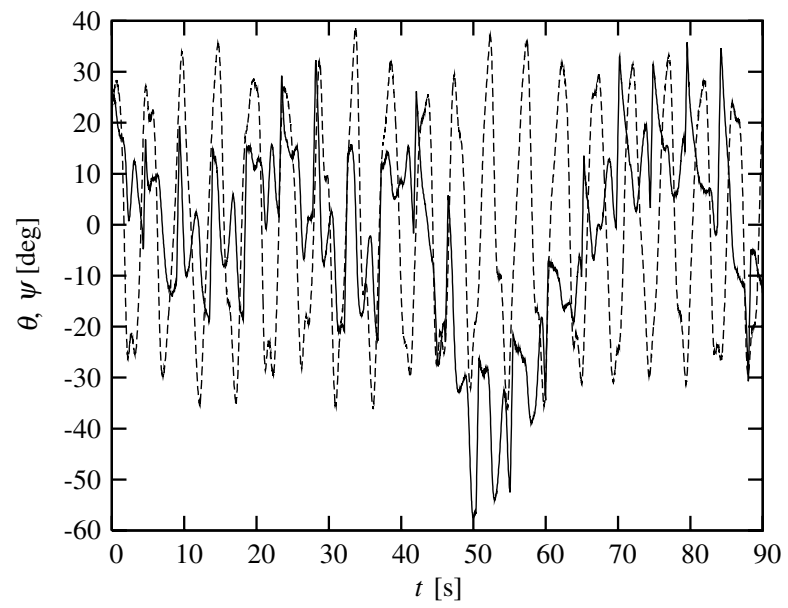

Fig. $10 R e=4.8 \times 10^{4}$. Time series of $\theta($ dashed line $)$ and $\psi($ solid line $)$ vs time $t$.

corresponds to the number of revolutions per second around the yaw axis. Almost the same plot is obtained for the power spectrum of $\theta$ (channel 2), reported in Fig. 8 for the same Reynolds number $R e=4.8 \times 10^{4}$. Spectra of $\theta$ and $\varphi$ look alike, except for some local (and small) differences. This is clearly due to the coupling between the angles $\varphi$ and $\theta$ caused by the joint geometry.

The power spectrum of $\psi$ (i.e., channel 3 purged from the uniform rotation around the yaw axis) is shown in Fig. 9. The same fundamental reduced frequency $k=5.3 \times 10^{-3}(f=0.215 \mathrm{~Hz})$ as in channel 1 and channel 2 is found together with its second and third harmonics. Compared to the preceding ones, however, this spectrum is very rich, especially at low frequencies. The presence of the latter imply long-period oscillations of the signal that are more visible in the time series reported in Fig. 10. Whereas $\theta$ (dashed line) is almost a sinusoidal wave, $\psi$ (solid line) features slow oscillations on top of the fundamental one at $k=5.3 \times 10^{-3}$. The presence of the same dominant frequency in all signals indicates the strong coupling between all of them. However, in the spectrum of $\psi$ the second and third harmonics are more visible than in the spectra of $\varphi$ and $\theta$. One might notice that the scale of Figs. 7 and 8 differs from that of Fig. 9 . This is due to the fact that the peaks in the spectra of $\varphi$ and $\theta$ are very sharp, and thus reach large values, whereas the peaks in the signal of $\psi$ are spread on larger bands of frequencies and are, therefore, lower.

The projection of the attractor from the phase space onto the $\varphi-\theta$ plane is shown in Fig. 11. Trajectories visit, approximately, a single loop, and the spin motion at $R e=4.8 \times 10^{4}$ is rather regular. It can be noticed that the trajectory appears more as a bundle than as a clear single orbit. This seems to be related to the particular configuration 


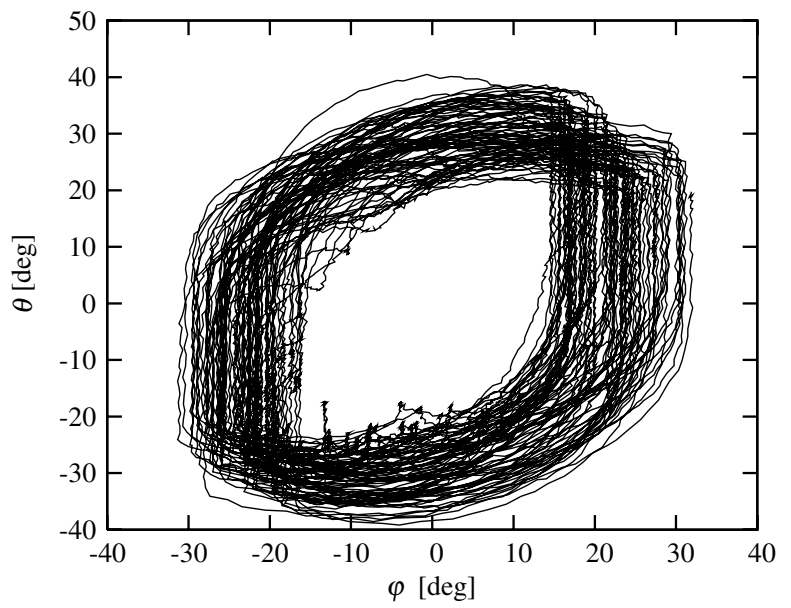

Fig. $11 R e=4.8 \times 10^{4}$. Phase portrait onto $\varphi-\theta$ plane (channel 1channel 2).

under investigation rather than to experimental noise or turbulence, even if the latter certainly provide a contribution. The effect of noise or turbulence, in fact, should be more pronounced at lower speeds, but by comparing all tests in basic configuration it can be concluded that the width of the "bundled orbit" remains roughly constant with Reynolds number (see tests in the following figures). Changes in the model configuration, on the contrary, show that the effects of noise or turbulence are within $4 \mathrm{deg}$ in the phase portrait (see, e.g., Fig. 27).

The fact that $\varphi$ remains almost constant every time it reaches its maximum or minimum (around -30 and $+30 \mathrm{deg}$ ) might look suspicious and one could think that the oscillations of $\varphi$ are limited by some physical constraints imposed by the universal joint or by its interaction with the model. This was excluded by visual inspection while the model was rotating and by the fact that in the worst-case scenario the limiting angles for $\varphi$ and $\theta$ are about $65 \mathrm{deg}$ (well above $30 \mathrm{deg}$ ). In addition, the vertical trajectories do not occur always at the same value of $\varphi$, as it would happen in case of constraints, but range between -32 and $-18 \mathrm{deg}$ for the lower values and between 14 and $30 \mathrm{deg}$ for the greater ones. It should be noticed, finally, that if any hitting occurred it would have resulted in sharp changes of the slope and not in almost straight lines.

The increase of Reynolds number to $R e=8 \times 10^{4}$ leads to a first bifurcation, as visible in Fig. 12. The first and highest peak still corresponds to the frequency of revolution around the yaw axis, $k=4.77 \times 10^{-3}$, but a new one appears at about $k=1.3 \times 10^{-2}$. Because, apparently, the ratio between these two values is not a simple rational number, the motion cannot be identified as periodic and is supposedly quasi-periodic. The presence of the additional higher frequency produces a more complex structure of the attractor, which is shown in Fig. 13. The main difference with the case at lower

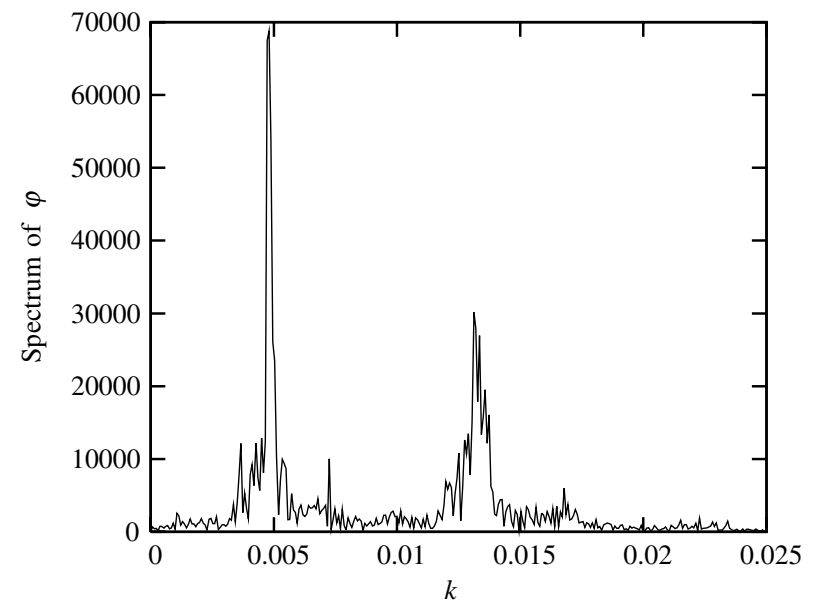

Fig. $12 R e=8 \times 10^{4}$. Power spectrum of $\varphi$ (channel 1$)$ vs the reduced frequency $k=f c / U_{\infty}$.

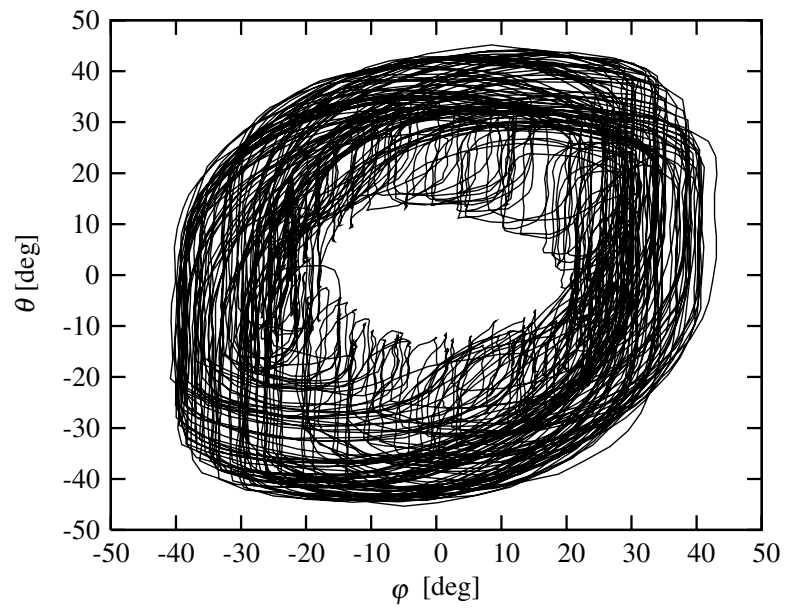

Fig. $13 R e=8 \times 10^{4}$. Phase portrait onto $\varphi-\theta$ plane (channel 1channel 2).

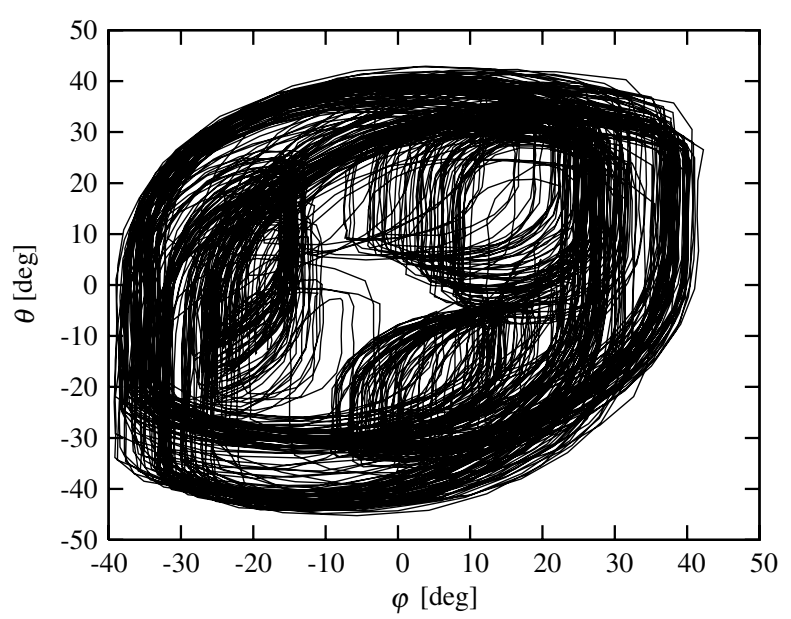

Fig. $14 R e=1.12 \times 10^{5}$. Phase portrait onto $\varphi-\theta$ plane (channel 1channel 2).

Reynolds number is the presence of smaller orbits within the bigger ones. The general shape of the larger orbits remains unchanged but they become slightly larger with $\varphi$ now ranging between -40 and $+40 \mathrm{deg}$, as opposed to $\pm 30 \mathrm{deg}$ previously observed. The inner region of the attractor is thus more visited than what happened in Fig. 11. It should be noticed that the smaller orbits arising in the central region have almost the same shape as the larger ones, a typical characteristic of fractals.

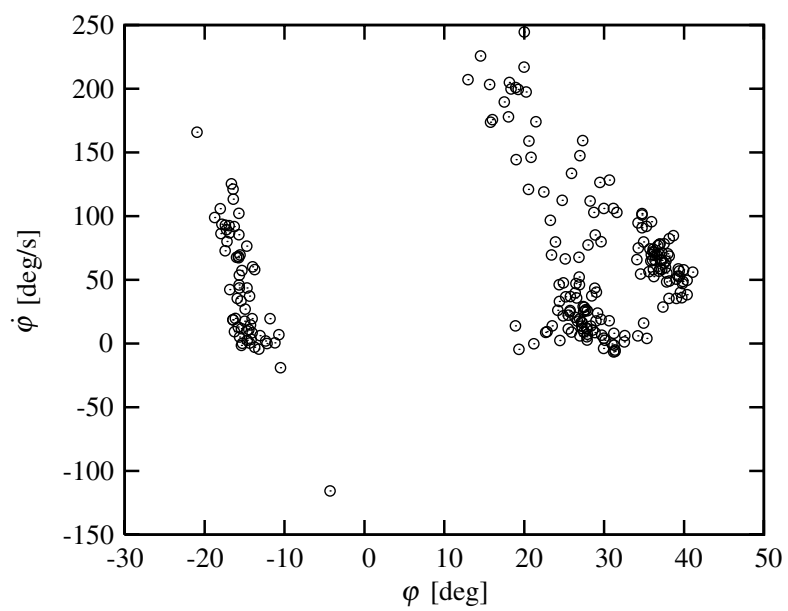

Fig. $15 R e=1.12 \times 10^{5}$. Poincaré section for $\theta=0$ and $\dot{\theta}>0$. 


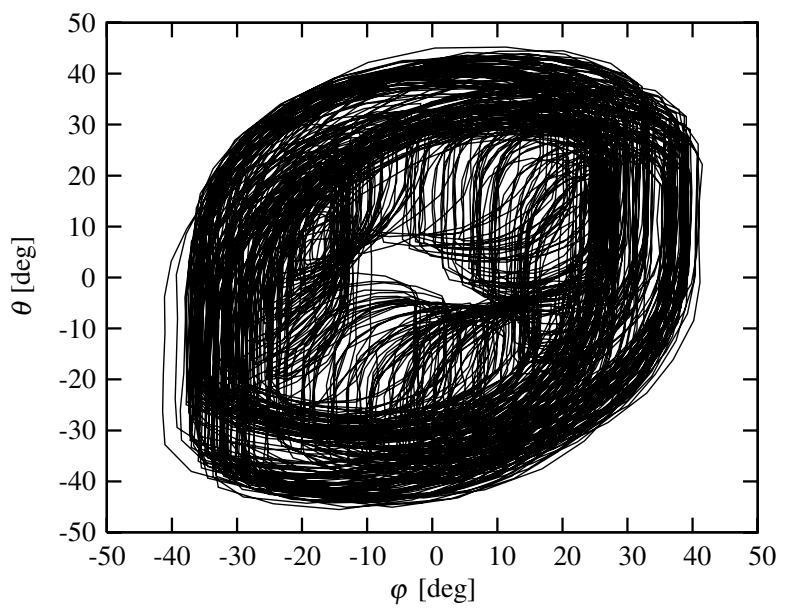

Fig. $16 R e=1.44 \times 10^{5}$. Phase portrait onto $\varphi-\theta$ plane (channel 1channel 2).

A further increase of the Reynolds number to $R e=1.12 \times 10^{5}$ carries along a more complex structure of the attractor projected onto the plane $\varphi-\theta$. In Fig. 14, two big trajectories seems to be associated with three small ones. This is due to the presence of four frequencies in the spectra, which are not reported but their values are plotted in Fig. 28. Because their ratio is still an apparently irrational number, the motion is believed to be quasi-periodic. More insights can be obtained from Poincaré sections. Here we perform a cut in the plane $(\theta=0, \dot{\theta}>0)$ and plot $\varphi$ vs its time derivative $\dot{\varphi}$. Figure 15 shows the presence of a region frequently visited by the attractor and another more rarely visited. The spatial correlation of the points in the map suggests the quasi-periodic nature of the motion, possibly developing toward chaos.

Figure 16 reports the projection of the attractor for $R e=1.44 \times 10^{5}$. The general trend associated with the increase of Reynolds number is confirmed. The center of the bigger trajectories is further filled with smaller ones, whereas the external trajectories remain unchanged. Even though 3 degrees of freedom are measured and available, we wanted to check if this complex structure of the attractor was contained in a single signal and could be unfolded in the embedding space. This experiment is shown in Fig. 17, where $\varphi(t+\Delta t)$ is plotted vs $\varphi(t)$ with $\Delta t=0.08 \mathrm{~s}$. Only one-tenth of the test is reported to make it more clear (a dark bundle would have been visible otherwise). The very same structure as in Fig. 16 is reproduced. In particular, small and large trajectories can be easily recognized and the same shape of the orbit repeats at different scales.

The general trend of a progressive increase in the complexity of the attractor as a function of the Reynolds number is confirmed also for $R e=1.63 \times 10^{5}$. Figure 18 shows the presence of four peaks in the

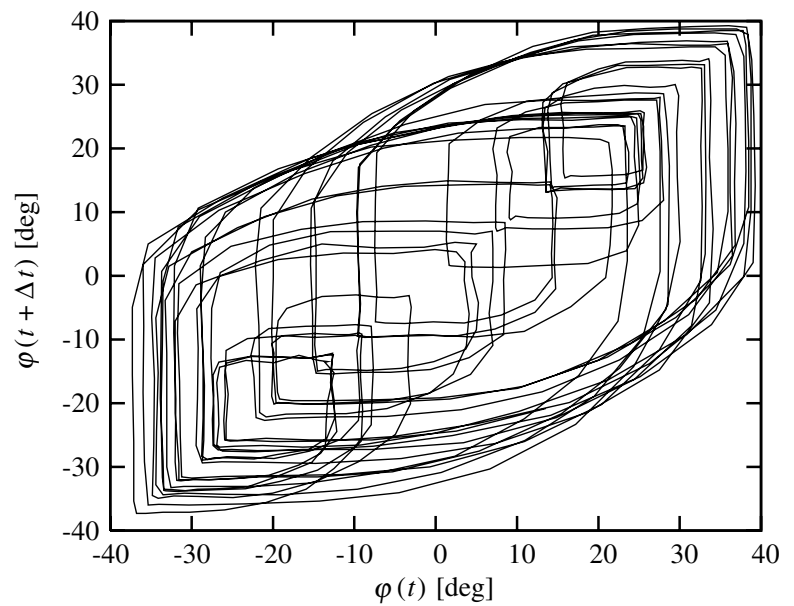

Fig. $17 R e=1.44 \times 10^{5}$. Projection of the attractor from the embedding space, $\Delta t=0.08 \mathrm{~s}$.

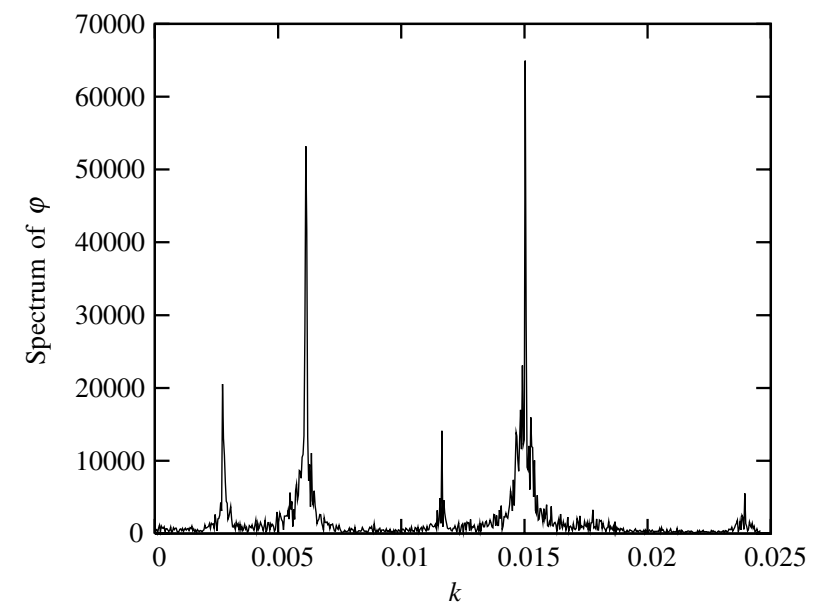

Fig. $18 R e=1.63 \times 10^{5}$. Power spectrum of $\varphi$ vs the reduced frequency $k=f c / U_{\infty}$.

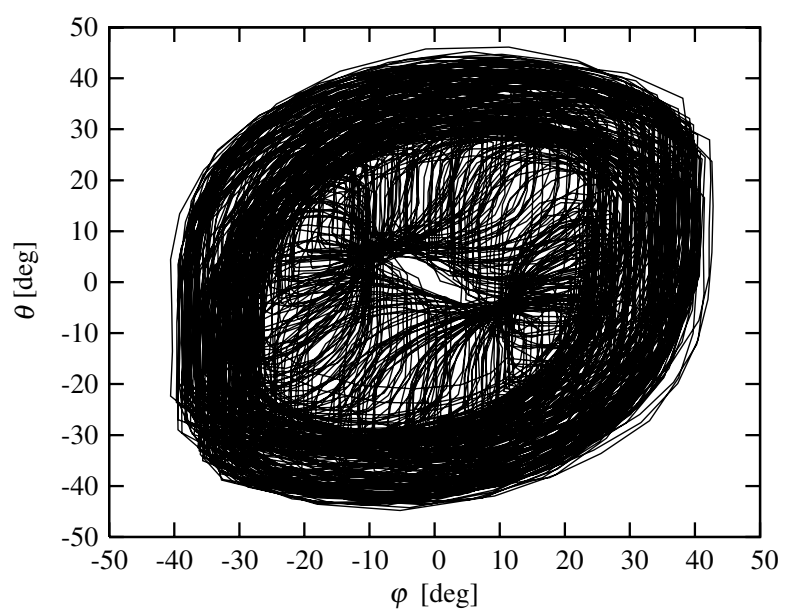

Fig. $19 R e=1.63 \times 10^{5}$. Phase portrait onto $\varphi-\theta$ plane (channel $1-$ channel 2).

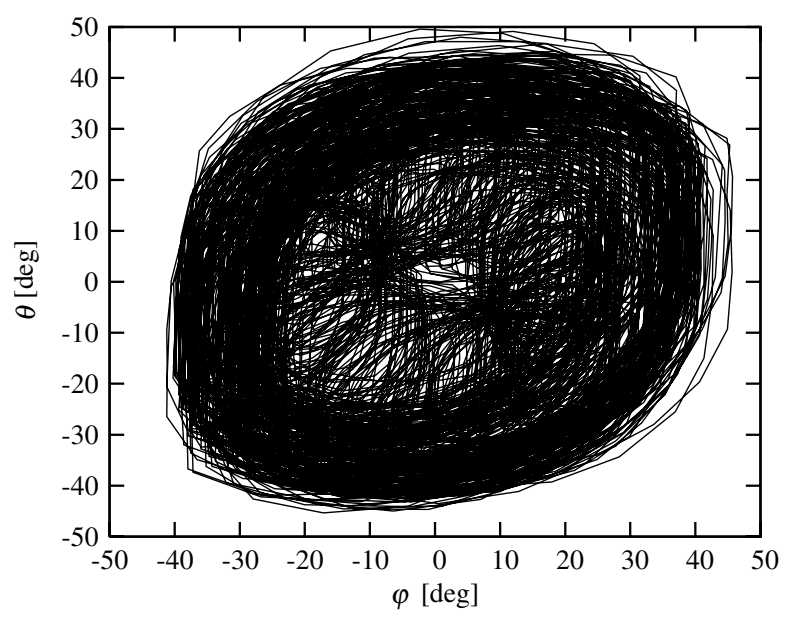

Fig. $20 R e=2 \times 10^{5}$. Phase portrait onto $\varphi-\theta$ plane. The internal region is completely filled.

spectrum, where the main one still corresponds to the number of revolutions per second around the yaw axis and the others are in irrational ratio with it. The quasi-periodic character of the motion is thus evident. A further evidence that corroborates the hypothesis of such a motion is the projection of the attractor, reported in Fig. 19. At $R e=1.63 \times 10^{5}$, the internal region is almost completely covered 


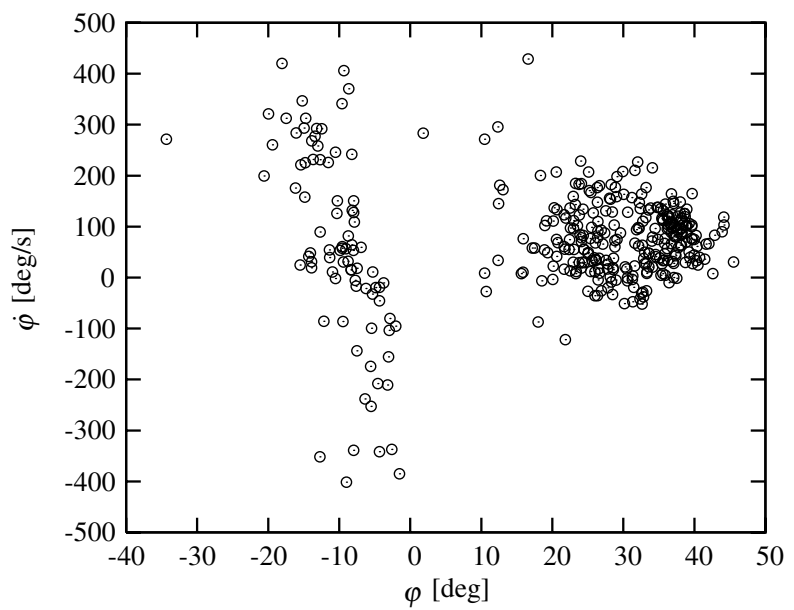

Fig. $21 R e=2 \times 10^{5}$. Poincaré section for $\theta=0$ and $\dot{\theta}>0$.

by the small orbits generated inside the bigger ones, with only one small region left unvisited in the center. It should be noticed that the external orbits remain unaffected by the increase of Reynolds number.

At $R e=2 \times 10^{5}$ (Fig. 20) the internal region of the attractor projection is, eventually, completely filled with the small orbits. However, this enhanced complexity of the attractor (compared to the cases at smaller Reynolds number previously reported) does not alter substantially the Poincaré section obtained for $\theta=0$ and $\dot{\theta}>0$. Figure 21 shows the presence of only two main regions visited by the attractor, as found at lower Reynolds number, but their shape does not seem to change considerably. The only difference is that now more points are visible for negative values of $\dot{\varphi}$.

\section{B. Modified Configurations}

The rather complex nature of the spin motion observed in basic configuration, particularly visible in the projection of the attractor onto the $\varphi-\theta$ plane and in the power spectra, raised the question whether the same complexity could be found also for modified configurations of the wind-tunnel model. Further tests were thus conducted to assess the influence of nose, wing geometry, tail planes, and their mutual interactions on the spin motion. In this section the following configurations are considered: 1) blunt nose, 2) leadingedge extensions on the wings, and 3) blunt nose + LEX. The analysis of the model motion without tail planes is reported in Sec. IV.C. The model with blunt nose and LEX is as reported in Fig. $\underline{6}$.

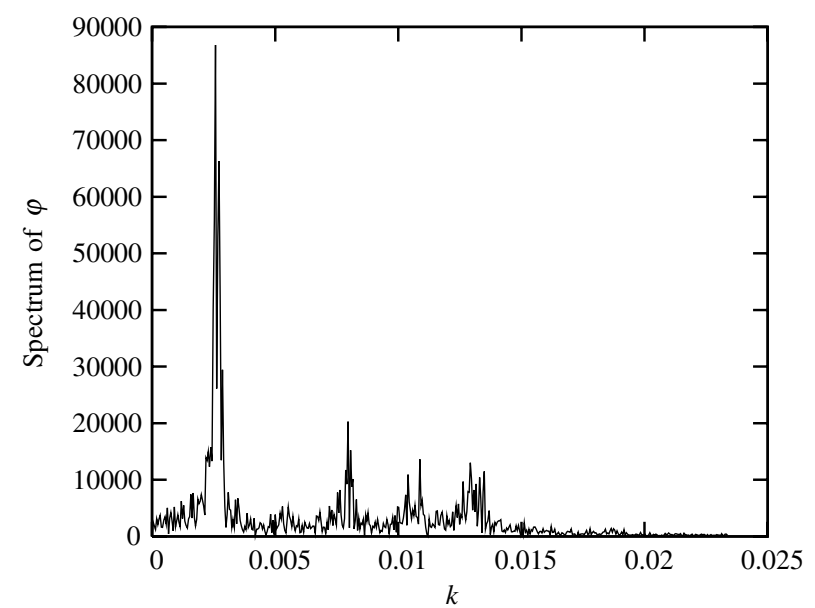

Fig. $22 R e=1.28 \times 10^{5}$, blunt-nose configuration. Power spectrum of $\varphi$ vs the reduced frequency $k=f c / U_{\infty}$.

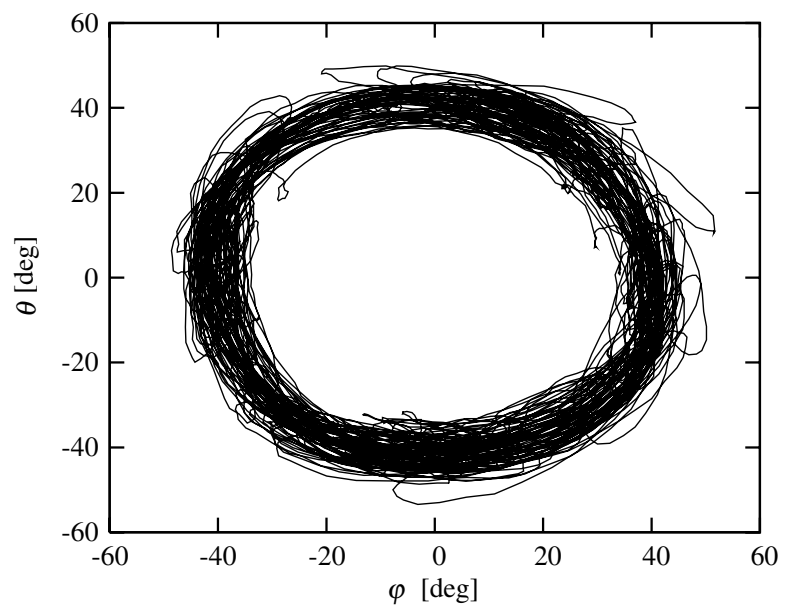

Fig. $23 R e=1.28 \times 10^{5}$, blunt-nose configuration. Phase portrait onto $\varphi-\theta$ plane.

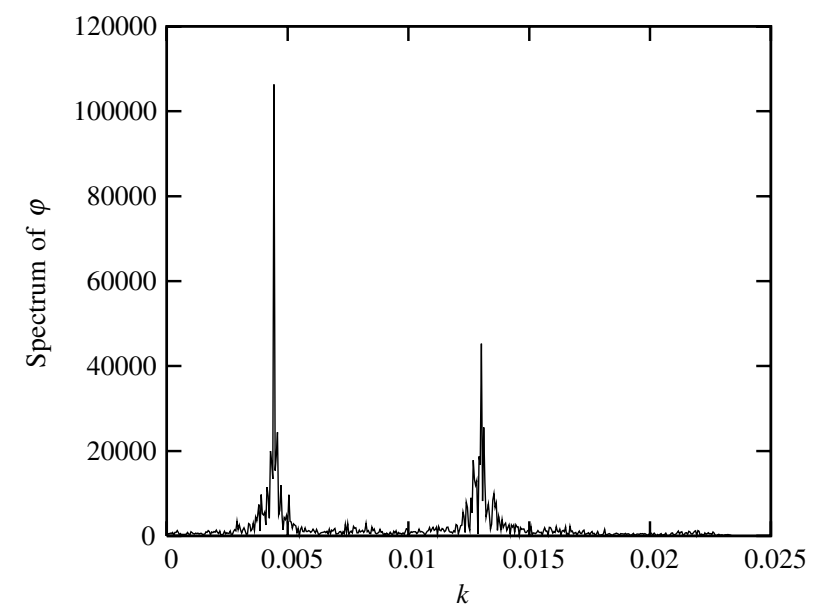

Fig. $24 R e=1.28 \times 10^{5}$, LEX configuration. Power spectrum of $\varphi$ vs the reduced frequency $k=f c / U_{\infty}$.

Results for the blunt-nose case obtained at $R e=1.28 \times 10^{5}$ are reported in Figs. 22 and 23 . In this configuration the wing is trapezoidal, as in Fig. $\underline{5}$, whereas the nose is as in Fig. $\underline{6}$. The power spectrum reveals the presence of four peaks. The first one is the frequency of revolution around the yaw axis, whereas the others are the third, fourth, and fifth harmonics. This is new compared to the previous spectra presented. Even though the spectrum is very rich, all frequencies are clearly in natural ratio, and thus the motion is periodic and not particularly complex. This is confirmed by the projection of the attractor, shown in Fig. 23, which features some big orbits confined in a relatively small region and visited together with some other smaller ones. It should be noticed that in this configuration the model was spinning in the opposite direction than in basic configuration. This excludes the possibility of a spin motion determined by residual swirl in the wind-tunnel flow and reinforces the hypothesis of a strong dominance of the fuselage through the influence of nose-vortices asymmetry.

The addition of LEX alone to the original conical nose produces the results in Figs. 24 and 25. The power spectrum shows only two very sharp peaks, where the first one is the usual frequency of revolution around the yaw axis and the second one is its third harmonics. The motion is thus much more regular than its counterpart in basic configuration, for which at the same Reynolds number $R e=1.28 \times 10^{5}$ four peaks in irrational ratios are found (see Fig. 28). The projection from the phase space, Fig. 25, features a system of large and small orbits, very similar in the topology to what was found for the basic configuration. The main difference, however, is that the presence of LEX excludes the possibility for $\varphi$ and $\theta$ of 


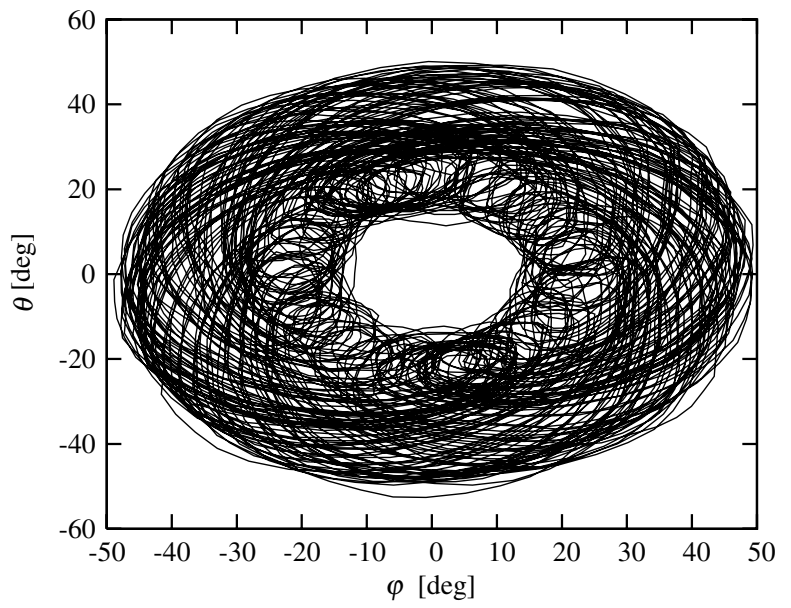

Fig. $25 R e=1.28 \times 10^{5}$, LEX configuration. Phase portrait onto $\varphi-\theta$ plane.

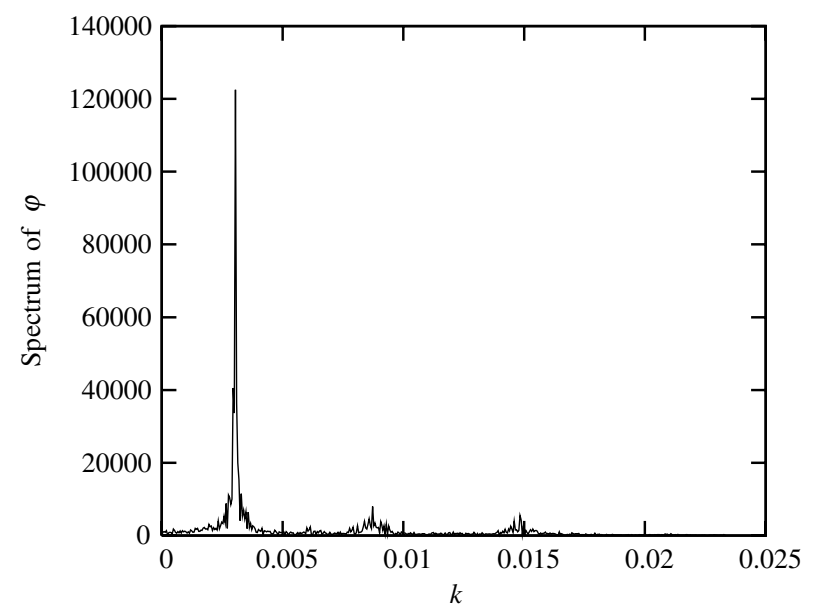

Fig. $26 R e=1.28 \times 10^{5}$, blunt nose + LEX configuration. Power spectrum of $\varphi$ vs the reduced frequency $k=f c / U_{\infty}$.

being simultaneously small and enlarges their ranges between \pm 50 deg. A reasonable interpretation of this behavior is that the addition of other surfaces (LEX) produces an increase in the roll damping, which does not allow the rise of further frequencies or the total covering of the central region of the projection. During all tests with LEX the model was spinning in the same direction as in basic configuration. This reconfirms that spin was dominated by the fuselage rather than the wing.

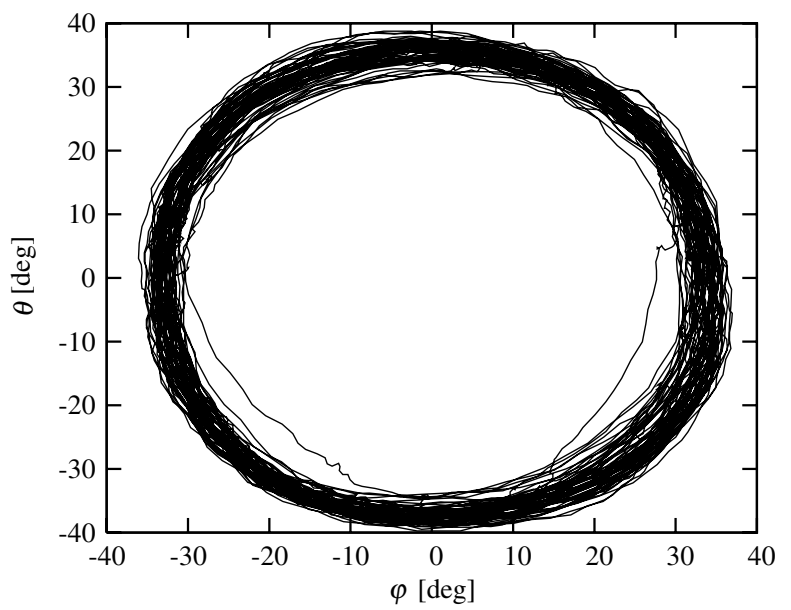

Fig. $27 R e=1.28 \times 10^{5}$, blunt nose + LEX configuration. Phase portrait onto $\varphi-\theta$ plane.
Because both blunt nose and LEX made the spin more regular, also their simultaneous effect was investigated. Figure 26 shows the power spectrum, with a major peak at $k=3.1 \times 10^{-3}$, corresponding to the frequency of spin around the yaw axis, and two very small peaks, which are its second and third harmonics. The motion is almost purely sinusoidal, because the amplitude of these harmonics is about 30 times smaller than the main frequency. This is confirmed by the projection of the phase portrait, Fig. 27. The trajectories are, by far, the closest to those of a pure periodic motion that could be observed in this campaign of experiments. The effects of blunt nose, which does not promote the formation of dangerous asymmetric vortices that could interfere with the tail planes producing strong yaw- and roll-exciting moments, and LEX, which by increasing pitch and roll damping stabilize the spin, together contribute to the regularization of the post-stall motion. The model in this configuration was spinning in the same direction as with blunt nose alone, i.e., opposite to the pointed nose. This confirms, once more, the fundamental role played by the fuselage compared to the wing. Another unique feature of this configuration is that only the left region of the resulting Poincaré section was visited.

\section{Influence of Tail Planes}

From the tests carried out in modified configurations, particularly with blunt nose, it seems that the aerodynamic influence of the fuselage geometry is a crucial factor for the onset of spin. This is mainly due to the yaw moments caused by the fact that nose vortices break down differently depending on whether the nose is a sharp or blunt cone. However, the nose and the fuselage might have also another affect on the excitation of spin. In fact, the vortices originating from the sharp-cone fuselage could interact with the tail planes, especially the horizontal one, causing asymmetric roll moments. This was not considered in the initial design of the model, but deserved further investigations, which have been carried out without the horizontal and/or vertical tail planes. It should be noticed that the model without tail planes was rebalanced to reduce possible alterations of the spin characteristics due to a modified mass distribution.

The model without the horizontal tail plane does not spin but oscillates in pitch only, at a very high angle of attack. Sometimes it starts to rotate in yaw direction, but stops very soon. The reason is, probably, that without the pitching moment due to the stabilizer, the angle of attack is very high, the nose vortices burst very close to the apex, and the asymmetrical loads are therefore too small to induce the spin motion.

The model without the vertical tail plane spins easily, even at low Reynolds numbers, and very similarly to the basic configuration. The motion is not very complex, probably due to the absence of the aerodynamic interaction between the nose vortices and the vertical plane.

Finally, without tail planes the model does not spin at all, confirming the important role played by their aerodynamic interaction with the vortices originating from the nose.

From these considerations it seems that the vertical tail plane does not essentially affect the spin, whereas the horizontal one plays an important role in the onset and in the maintenance of the spin motion. This hypothesis is supported by the fact that (incipient) spin motion without the vertical tail plane is not so different from the one in basic configuration. Two possible explanations of this behavior could be given. According to the first one, probably an asymmetric distribution of the aerodynamic load on the horizontal tail plane, due to the interaction with the wing and fuselage wakes, can produce a roll moment not damped by the wings. The induced roll motion causes a yaw moment because of the different drag on the two wings, eventually leading to spin.

The other possible explanation is that the presence of the stabilizer contributes to reduce the angle of attack of the model. At low incidence, the nose vortices are not yet broken down and their asymmetry could produce a yaw moment strong enough to spin the airplane. 
To check the validity of this suppositions, it was decided to attempt smoke flow visualization for the basic configuration. The tail and the pointed nose were particularly investigated as they were supposed to be the most critical for the spin motion.

During flow visualization on the tail the model was kept fixed at the two pairs of angles $(\varphi, \theta)$ corresponding to a sudden change in the phase portrait seen in all plots of the basic configuration, namely, the pairs ( $-35 \mathrm{deg},-35 \mathrm{deg})$ and ( $35 \mathrm{deg}, 35 \mathrm{deg})$. In these conditions it was found that the flow over the horizontal tail plane is attached only on half of it, whereas it is separated on the other one due to the wakes of the wing and the fuselage. This asymmetry provokes inevitable asymmetric aerodynamic moments accompanied by sudden changes in the trajectories, particularly visible on the projections of the attractor.

The other set of flow visualizations was carried out over the nose, at two angles of attack, $\alpha=40 \mathrm{deg}$ and $\alpha=70 \mathrm{deg}$. At low incidence the nose vortices are effective (not broken down yet), and thus they influence the motion either via their asymmetric breakdown or via their interaction with the tail. On the contrary, at large incidence $(\alpha=70 \mathrm{deg}$ ) vortex breakdown has already taken place and their aerodynamic influence on spin is negligible.

\section{Is the Motion in Basic Configuration on a Route to Chaos?}

It appears from the results showed for the model in basic configuration that the motion has both regular and quasi-periodic aspects, in a possible route toward chaos.

It has been found that for increasing Reynolds number the motion becomes more complex and the frequencies detected are not in simple ratios. Figure 28 summarizes the dependence of the number of frequencies and their behavior as a function of the Reynolds number.

At low Reynolds number, only the primary frequency corresponding to the number of revolutions per second around the yaw axis is present. Two successive bifurcations take place, at about $R e=5.6 \times 10^{4}$ and $R e=8.7 \times 10^{4}$. Within the range of freestream Reynolds numbers that was possible to test (the limit on the maximum speed is dictated by the wind-tunnel fan), no further bifurcations occurred and no more than four frequencies could be detected in the signals. These frequencies seem not to be harmonics as their ratio with the primary one is not a rational number. It should be noticed that the reduced frequency $k$ is a slightly increasing function of the Reynolds number only for the primary and second frequencies, whereas the other two frequencies that appear for $R e>$ $8 \times 10^{4}$ are almost constant or slightly decreasing with Reynolds number. The overall change in the reduced frequencies is, however, quite small.

Figure 28 provides also some information related to the aerodynamics of the forebody. The primary and second frequencies feature an apparently anomalous behavior for Reynolds number greater than about $1.8 \times 10^{5}$, with a sort of upward step. The corresponding Reynolds number based on the diameter is about $R e_{d}=1.7 \times 10^{5}$, which is quite close to the critical Reynolds number for a cylinder $R e_{d} \approx 2 \times 10^{5}$. Therefore, the strong increase in the reduced frequency might be due to the aerodynamics of the forebody.

The features summarized in Fig. 28, with the appearance of further frequencies in apparently irrational ratios while increasing a parameter (Reynolds number), are typical of quasi-periodic systems. However, in their route to chaos many systems experience the quasiperiodic stage right before the complete evolution to chaos. Therefore, even though a chaotic behavior has not been observed during these experiments, the question of whether the system depending on the parameter Reynolds number is on its route to chaos is legitimate.

The answer cannot be "no" because some evidence of typical behaviors of systems evolving toward chaos were found. The structure of the attractor, for instance, becomes more and more complex as the Reynolds number increases, clearly resembling a bundle. Moreover, quite regular trajectories are found at low Reynolds number, but as soon as the second frequency appears,

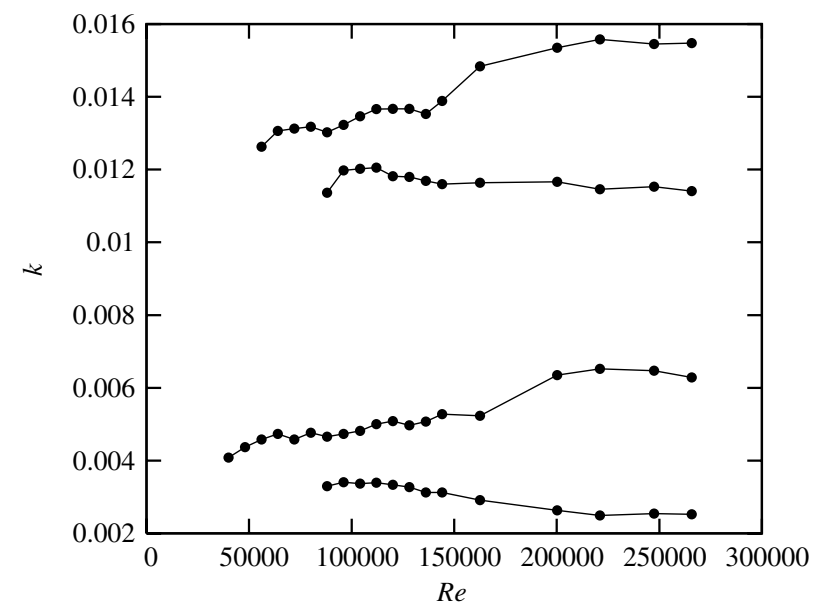

Fig. 28 Reduced frequency vs Reynolds number.

smaller loops show up in the projection of the attractor. Incidentally, the shape of these smaller loops is the same as the bigger ones, in a fashion similar to what happens on different scales for fractals. To show this more clearly, Fig. 29 reports only one-tenth of the plot showed in Fig. 13. The smaller orbits are similar to the bigger ones, exhibiting their fractal nature, but no further smaller scales exist. As seen in the section devoted to the results, Sec. IV, at low Reynolds number only one big trajectory can be recognized, whereas the increase of Reynolds number leads to the appearance of small loops, progressively covering all the internal region for high values of the Reynolds number.

Some other insights regarding the possibility for the system of being in a route to chaos have been obtained from some Poincaré maps. In those sections it was possible to identify "clouds" of points, located in particular regions of the space and thus with some spatial structure (see Sec. IV). A further postprocessing of the data was then carried out to understand if there is any recognizable pattern in the way the attractor switches from one region to the other and if the motion exhibits sensitivity to initial conditions. From two tests in the same conditions (basic configuration, $R e=1.28 \times 10^{5}$ ) the sequence of passages from one to the other cloud was detected by marking with " 1 " the presence in the most crowded region (righthand cloud) and with " 0 " the presence in the other one. Results are here reported:

Sequence 1 (227 intersections with the plane $\theta=0$ and $\dot{\theta}>0$ ): 11 01110111110111011011101111101101110111111 11011101111011111011101111101110111111111 01110111011011111011111011111011011101110 11101110111011111111011110110111011101111 10111011011111011101110111011011011101110 11111011110111011111

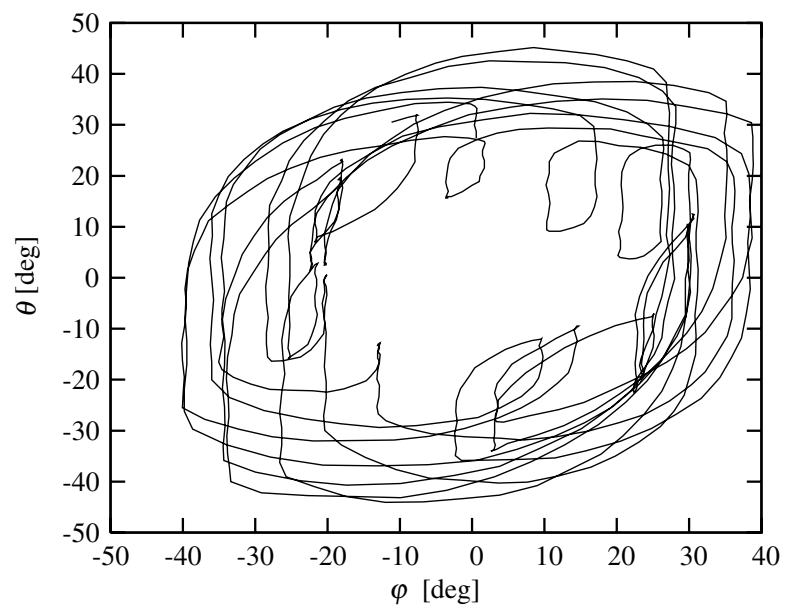

Fig. $29 R e=8 \times 10^{4}$. Partial phase portrait $(1 / 10$ of the total test) onto $\psi-\theta$ plane. 
Sequence 2 (237 intersections with the plane $\theta=0$ and $\dot{\theta}>0$ ): 10 11101111111111011101111101110110110111011 10110111011101110111011011101101111101110 11011011011111111111011011101111101110111 01111110110111011101111101111011101101110 11011101110111110110111011111011101111111 110111011101101111101101110111

The fact that there is no definitive sequence repeating itself in each test suggests that the motion in basic configuration is quite unpredictable and certainly not periodic. On the other hand, because a similar sequence cannot be observed when comparing the two tests, it can also be concluded that the spin motion in basic configuration is sensitive to initial conditions. The latter is typical of nonlinear and chaotic systems.

The question of whether the motion is quasi-periodic or chaotic can thus be answered. The motion observed in these experiments is not chaotic, but might be on its route to chaos. The power spectra are typical of quasi-periodic systems, but the attractor shows characteristics of systems developing toward chaos, especially the impossibility to predict which region of the phase space will be visited by the attractor (the same happens for Lorenz attractor) and sensitivity to initial conditions.

A simple explanation could be that at higher Reynolds numbers chaos might appear, but this state was not achieved in the present experiments due to the physical limitations of the wind-tunnel capabilities (maximum Reynolds number reachable). A different, and more physical, explanation might be found in the role of the aerodynamic forces. In fact, they are the exciting source of the spin motion, but when they do not excite they damp it, thus inhibiting the development of further higher frequencies and/or lower subharmonics.

\section{Conclusions}

The model of an airplane designed to feature the worst possible spin has been tested in a conventional wind tunnel.

In basic configuration (conical nose and trapezoidal wing) the motion can be classified as quasi-periodic. The number of frequencies detected in the power spectra increases with the Reynolds number from one, corresponding to the number of revolution around the yaw axis, to four. Two successive bifurcations occur, and the ratio between the frequencies so originated is not a simple (rational) number. This suggests the presence of a quasiperiodic motion, possibly on its route to chaos. Further analyses involving projection of the attractor and Poincare sections have thus been carried out. Even though a clear chaotic behavior cannot be assessed, many characteristics typical of chaotic systems have been found. The complex structure of the attractor, and especially the unpredictability of the regions occupied by the attractor in the phase space while switching from one region to another (as for Lorenz attractor), could leave room for a possible evolution to chaos. However, only the initial aspects of this route have been observed, and a true fractal nature of the motion is not established.

Tests in modified configurations showed that the original conical nose is largely responsible for the spin motion and has a negative effect on it. On the contrary, blunt nose and leading-edge extensions (LEX) ensure a much more regular behavior. Not only did they promote an almost purely periodic motion, but they also increased by a factor of three the Reynolds number necessary for the onset of spin. By removing one or both tail planes it has been verified that the horizontal one plays an essential role in the incipient spin motion, because it seems to be an almost necessary condition for spin to occur.

Flow visualizations supported the hypothesis about the aerodynamic interactions between the nose vortices and the tail planes. Thus, although the original idea motivating this study was the possible coupling between the wing and fuselage spins, it seems from the tests that the main coupling is between the nose and the tail planes.

This study, by correlating the qualitative dynamic behavior of spin characteristics with changes in geometry, provides also direct guidance on potential ways of modifying the spin features of an aircraft.

\section{References}

[1] Anon., Flight Training Handbook, Federal Aviation Agency, AC 6121, Washington, DC, 1965.

[2] DeLacerda, F. G., Facts About Spins, Iowa State Press, Ames, IA, 2002.

[3] Kimberlin, R. D., Flight Testing of Fixed-Wing Aircraft, AIAA Education Series, AIAA, Reston, VA, 2003.

[4] Chambers, J. R., and Bowman, J. S., Jr., "Recent Experience with Techniques for Prediction of Spin Characteristics of Fighter Aircraft," Journal of Aircraft, Vol. 8, No. 7, 1971, pp. 548-553.

[5] Stough, H. P., DiCarlo, D. J., and Patton, J. M., "Flight Investigation of Stall, Spin, and Recovery Characteristics of a Low-Wing, SingleEngine, T-Tail Light Airplane," NASA Technical Rept. NASA TR2427, 1985

[6] Fremaux, C. M., "Spin-Tunnel Investigations of a 1/28-Scale Model of the NASA F-18 High Alpha Research Vehicle (HARV) with and Without Vertical Tails," NASA Contractor Rept. NASA-CR-201687, 1997.

[7] Croom, M., Kenney, H., and Murri, D., "Research on the F/A-18E/F Using a 22\%-Dynamically-Scaled Drop Model," AIAA Paper 20003913, 2000.

[8] Stough, H. P., Patton, J. M., Jr., and Sliwa, S. M., "Flight Investigation of the Effect of Tail Configuration on Stall, Spin, and Recovery Characteristics of a Low-Wing General Aviation Research Airplane," NASA Technical Rept. TP-2644, 1987.

[9] Tischler, M. B., and Barlow, B., "Determination of the Spin and Recovery Characteristics of a General Aviation Design," Journal of Aircraft, Vol. 18, No. 4, 1981, pp. 238-244.

[10] Carroll, J. V., and Mehra, R. K., "Bifurcation Analysis of Nonlinear Aircraft Dynamics," Journal of Guidance and Control, Vol. 5, No. 5, 1982, pp. 529-536.

[11] Zagayonov, G. I., and Goman, M. G., "Bifurcation Analysis of Critical Aircraft Flight Regimes," ICAS Paper 84-4.2.1, 1984.

[12] Goman, M. G., Zagayonov, G. I., and Khramtsovsky, A. V., "Application of Bifurcation Methods to Nonlinear Flight Dynamics Problems," Progress in Aerospace Sciences, Vol. 33, No. 9, Sept. 1997, pp. 539-586.

[13] Guicheteau, P., "Non-Linear Flight Dynamics," Non-Linear Dynamics and Chaos, AGARD Lecture Series no. 191, paper no. 5, AGARD, Neuilly sur Seine, France, 1993.

[14] Guicheteau, P., "Stability Analysis Through Bifurcation Theory (1 and 2)," Non-Linear Dynamics and Chaos, AGARD Lecture Series no. 191, paper no. 4, AGARD, Neuilly sur Seine, France, 1993.

[15] Lowenberg, M. H., "Bifurcation Analysis of Multiple-Attractor Flight Dynamics," Philosophical Transactions of the Royal Society of London, Vol. 356, No. 1745, Oct. 1998, pp. 2297-2319.

[16] Davidenko, D. F., "On a New Method of Numerical Solution of Systems of Nonlinear Equations," Doklady Akademii Nauk SSSR, Vol. 88, No. 4, 1953, pp. 601-602 (in Russian).

[17] Anglin, E. L., and Scher, S. H., "Analytical Study of Aircraft Developed Spins and Determination of Moments Required for Satisfactory Spin Recovery," NASA Technical Rept. TN D-2181, 1964.

[18] Taylor, L. W., Jr.,, and Miller, G. K., Jr., "An Analytical Technique for the Analysis of Airplane Spin Entry and Recovery," AIAA Paper 820243, 1982.

[19] Young, J. W., and Adams, W. M., Jr., "Analytical Prediction of Aircraft Spin Characteristics and Analysis of Spin Recovery," AIAA Paper 72985, 1972.

[20] Jaramillo, P. T., "An Analysis of Falling Leaf Suppression Strategies for the F/A-18D," AIAA Paper 96-3370, 1996.

[21] Lee, D. C., and Nagati, M. G., "Angular Momentum Control in Nonlinear Flight," Journal of Aircraft, Vol. 37, No. 3, 2000, pp. 448453.

[22] Lee, D. C., and Nagati, M. G., "Momentum Vector Control for Spin Recovery," Journal of Aircraft, Vol. 41, No. 6, 2004, pp. 1414-1423.

[23] Gránásy, P., Sørensen, C. B., Mosekilde, E., and Thomasson, P. G., "Non-Linear Flight Dynamics at High Angles-of-Attack," The Aeronautical Journal, Vol. 102, No. 1016, June-July 1998, pp. 337 343.

[24] Cao, J., Garrett, F., Jr., Hoffman, E., and Stalford, H., "Analytical Aerodynamic Model of a High Alpha Research Vehicle Wind-Tunnel Model," NASA CR 187469, 1990.

[25] Planeaux, J. B., and McDonnell, R. J., "Thrust Contributions to the Spin Characteristics of a Model Fighter Aircraft," AIAA Paper 1991-2887, 1991. 
[26] Tobak, M., and Chapman, G. T., "Nonlinear Problems in Flight Dynamics Involving Aerodynamic Bifurcations," AGARD CP 386, 1985.

[27] Goman, M. G., and Khramtsovsky, A. V., "Application of Continuation and Bifurcation Methods to the Design of Control Systems," Philosophical Transactions of the Royal Society of London, Vol. 356, No. 1745 , 1998, pp. 2277-2295.

[28] Raghavendra, P. K., Sahai, T., Kumar, P. A., Chauhan, M., and Ananthkrishnan, N., "Aircraft Spin Recovery, with and Without Thrust Vectoring, Using Nonlinear Dynamic Inversion," Journal of Aircraft, Vol. 42, No. 6, 2005, pp. 1492-1503.

[29] Mena, S., "Dalla vite al caos?," M.S. Thesis, Politecnico di Milano, Milan, 1994 (in Italian).

[30] Hui, W. H., and Tobak, M., "Bifurcation Theory Applied to Aircraft Motions," AGARD CP 386, 1985.

[31] Nelson, R. C., "Unsteady Aerodynamics of Slender Wings," AGARD R 776, 1991.

[32] Chambers, J. R., Anglin, E. L., and Bowman, J. S. J., "Effects of a Pointed Nose on Spin Characteristics of a Fighter Airplane Model Including Correlation with Theoretical Calculations," NASA TN D5921, 1970.

[33] Skow, A. M., Titiriga, A. J., and Moore, W. A., "Forebody/Wing Vortex Interactions and Their Influence on Departure and Spin Resistance," AGARD CP 247, 1978.

[34] Orlik-Rückemann, K. J., "Unsteady Aerodynamics and Dynamical Stability at High Angles of Attack," AGARD LS 121, 1982.
[35] Erickson, G. E., and Gilbert, W. P., "Experimental Investigation of Forebody and Wing Leading-Edge Vortex Interaction at High Angles of Attack," AGARD CP 342, 1983.

[36] Skow, A. M., and Peake, D. J., "Control of the Forebody Vortex Orientation by Asymmetric Air Injection: (Part A) Application to Enhance Departure/Spin Recovery of Fighter Aircraft and (Part B) Details of the Flow Structure," AGARD R 711, 1983.

[37] Malcom, G. N., "Forebody Vortex Control," AGARD R 776, 1991.

[38] Tuffilaro, N. B., Abbott, T., and Reilly, J., An Experimental Approach to Nonlinear Dynamics and Chaos, Addison Wesley, Reading, MA, 1992.

[39] Bergé, P., Pomeau, Y., and Vidal, C., L'ordre dans le chaos, Hermann, Paris, 1984.

[40] Abarbanel, H. D. I., Analysis of Observed Chaotic Data, SpringerVerlag, New York, 1996.

[41] Devaney, R. L., An Introduction to Chaotic Dynamical Systems, Benjamin/Cummings, Menlo Park, CA, 1986.

[42] Ott, E., "Strange Attractors and Chaotic Motions of Dynamical Systems," Reviews of Modern Physics, Vol. 53, No. 4, 1981, pp. 655671.

[43] Ott, E., Sauer, T., Gluckman, B. J., Grebogi, C., and Schiff, S., "Extracting Unstable Periodic Orbits from Chaotic Time Series Data," Physical Review, Vol. 55, No. 5, 1997, pp. 5398-5417.

[44] Takens, F., "Detecting Strange Attractors in Turbulence," Dynamical Systems and Turbulence, Warwick 1980, edited by D. Rand and L. S. Young, Springer-Verlag, Berlin, 1981, p. 366. 